\title{
Measles in a Neonatal Unit during Epidemic Period in 2011
}

\author{
Josefina Marquez ${ }^{1}$, Laura Acosta ${ }^{1}$, Manuel Fernandez ${ }^{2}$ \\ ${ }^{1}$ Neonatal Intensive Care Unit, Pediatric Departament, Virgen de Valme University Hospital, Seville, Spain; ${ }^{2}$ Pediatric Investigation \\ Unit, Pediatric Departament, Virgen de Valme University Hospital, Seville, Spain. \\ Email: drlolo13@hotmail.com
}

Received July 21 ${ }^{\text {st }}$, 2012; revised August 23 ${ }^{\text {rd }}, 2012$; accepted September $25^{\text {th }}, 2012$

\begin{abstract}
The incident of the measles has diminished in the last decades thanks to the widespread use of the vaccine in The United States and Europe. Nevertheless, recently we are seeing new epidemics of measles due to the lack of coverage in some social areas. Measles during pregnancy is associated with high maternal morbidity and mortality, being a frequent cause of abortion in preterm deliveries. We report two cases of measles attended in the Unit of Neonatology held coinciding with the epidemic lived in this area during the last year.
\end{abstract}

Keywords: Congenital Measles; Neonatal Measles; Maternal Morbidity; Vaccination; Extremely Low Birth Weight Newborn

\section{Introduction}

Measles is a highly contagious infectious disease with high mortality rate un the age group from 0 to 4 years old. In 2003, WHO estimated that measles caused around 530,000 deaths worldwide. The stages in the process of reducing measles deaths have strengthened efforts to improve understanding of the epidemiology of this disease and reduce its incidence.

It is caused by a virus of the Paramyxoviridae family and it clinically describes two stages. The first is called the prodromal phase, pre-exanthematic or cold, accompanied by fever of $38.5^{\circ} \mathrm{C}$ to $40^{\circ} \mathrm{C}$ and symptoms of upper airway such as cough, coryza and conjunctivitis. The second phase, called exanthematous or eruptive, begins the third or fourth day and it appears a characteristic rash with brown is red spots starting on the face and them generalize from 4 to 7 days. The transmission period begins when the clinical manifestations are not yet evident occurs by droplets expelled by the respiratory system when coughing or sneezing. These come in contact with the conjunctiva or upper respiratory tract of susceptible people. Before the onset of symptoms exists an incubation period about 14 days .

There is no specific treatment for measles except symptomatic. Measles gamma globulin can be administered intramuscularly in the first seven days after contact $(0.25$ $\mathrm{ml} / \mathrm{kg}$ ) in groups of children under 2 years old, old people and pregnant women. Intended for those who have been exposed to contact and have no immunity to measles.

As preventive measures, apart from the mandatory spe- cific vaccination, we know that the virus remains active for two hours in the enviroment. In this regard it is important to ventilate the room, disinfect surfaces, avoid close contact with known cases and measurements of respiratory isolation.

During the period before the implementation of systematic programs of vaccination in our setting, most cases of measles infection detected occurred in the early years of life, so that the vast majority of individuals suffering from the disease before reach adulthood. Due to these circumstances, the emergence of infectious measles was extremely rare in pregnant women. Since the eighties, the implementation of systematic and universal vaccination of the population caused a change in the epidemiology of the disease [1]. The cases in the first years of life ended up being rare. However, due to the complete coverage of vaccination and to judgments derived from the vaccine would increase the number of infected adults who had not received prophylactic vaccine. Thus, as women of childbearing age are at potential risk of measles infection during these outbreaks [2-4].

After some years with low incidence rates, from the year 2011 has been produced in our area a significant increase that has caused an epidemic of measles. In the period 2011-2012 have been diagnosed a total of 139 children aged 3 days to 14 years, of which 77 (55\%) were under 15 months old and therefore should be vaccinated at that time. The total number of cases identified in our hospital has increased to 351 individuals, of whom 82 were women of childbearing age (16 - 49 years). In these circumstances, a group of unimmunized women have been 
exposed to measles during pregnancy, which is associated with a high incidence of maternal morbidity and perinatal mortality [2-5].

We report two cases of measles seen at the Neonatal Unit of Hospital Universitario Virgen de Valme held coinciding with the outbreak lived in this area during 2011.

\subsection{Case 1: Measles Congenital}

This is a mother of 29 year old with two previous pregnancies. This is a pregnancy that presents clinically controlled with an episode of measles at 26 weeks of gestation. Maternal lesions appeared four days before the birth of an infant of 26 weeks' gestation and 995 grams in weight. After delivery was necessary implementation of resuscitation with endotracheal intubation and surfactant administration. On admission showed signs of immaturity, auscultation with acceptable ventilation and no murmurs. Abdomen normal. Palate and clavicles intact. Male external genitalia. No skin lesions were visualized. Isolation measures were established in the neonatal unit at the mother for her condition and sent samples for newborn screening for measles. During the first 32 hours of life, the infant remained connected to mechanical ventilation with hemodynamic and respiratory stability. He then presented a sudden worsening, with severe desaturations despite optimized ventilatory therapy and endotracheal tube massive bleeding. Were initiated advanced resuscitation for 20 minutes, which proved ineffective. The infant died after 48 hours of life. Study serology: IgM negative. PCR viral study: positive urine sample and Pharyngeal exudate.

\subsection{Case 2: Measles Neonatal}

Mother is a 25-year-old primigravida with a history of epilepsy treated with valproic acid and lorazepam. The baby was the result of spontaneous preterm delivery of 36 weeks gestational age, low weight $(2.280 \mathrm{~kg})$ and Apgar 9/10. At birth required admission for prematurity, remaining in the neonatal unit for 7 days. At 27 days requery in the emergency department for mild fever and feed refusal of 24 hours duration. Refer contact with various family members affected by measles, his mother presented a generalized rash the week before admission and can not remember having received vaccination against measles. Exploration Activity: 2.780 kg weight, axillary temperature $38^{\circ} \mathrm{C}$. Acceptable condition. Normal color of skin and mucous membranes with generalized morbilliform rash and no petechiae. Seromucous rhinorrhea. Conjunctival purlenta secretion. Cardiopulmonary examination and abdomen normal. On admission gamma globulin is administered iv and requesting additional tests found positive IgM serology for measles nasopharyngeal and serum. Is isolated by PCR of measles virus RNA in urine. In the other tests found no other findings of interest. At 8 days after admission the patient was discharged without complications.

\section{Discussion}

We currently have limited literature on the occurrence of measles during pregnancy, let alone during labor. Congenital measles includes cases where the rash is present at birth and also those infections acquired in utero in which the rash appears in the first ten days of life. The disease spectrum is wide, from mild clinical manifestations to fatal forms coming to kill. In the case of preterm infants prognosis is worse with increased mortality [1,6,7], as in the case presented. To confirm the diagnosis, in both cases were conducted and serological study of viral PCR in urine and nasopharyngeal swab. In our first case, the determination of Ig $\mathrm{M}$ was negative because positivized usually within 3 days into the rash, clinic not yet seen in our patient. Measles and neonatal appears in the first month of life not because of disease transmission during the period around birth. The isolation and preventive measures adopted at the neonatal unit were identical in both cases. Other parents of newborns admitted to the unit were informed and asked for the immune status of mothers against measles. The children of mothers who had not been vaccinated or had not previously had the disease; immune globulin was administered im, without registering any type of incident.

The epidemic experienced in the past year shows the importance of maintaining a correct and constant immunization strategy in the population, with particular emphasis on the immunity of children, adolescents and young adults who were not vaccinated at the time. Thus, we reduce the incidence of measles in women of child-bearing potential for future epidemics.

\section{Conclusions}

Measles is a disease that has not yet been eradicatd in the world, but thanks to vaccination campaigns have been reduced significantly the number of cases in the past two decades.

The epidemic experienced in 2011 shows the importance of maintaining a correct and constant immunization strategy in the population, with particular emphasis on the immunity of children, adolescents and young adults who were not vaccinated at the time.

The widespread use of vaccines involves a decrease in the incidence of measles in young women of childbearing age, who can transmit the disease uring pregnancy, and the risks that this disease entails in newborns.

\section{REFERENCES}

[1] H. Go, K. Hashimoto, T. Imamura, et al., “An Extremely 
Low Body Weight Infant Born to a Mother with Measles,” Journal of Perinatology, Vol. 30, No. 2, 2010, pp. 146-148. doi:10.1038/jp.2009.111

[2] M. Elamin and H. M. Albar, "Meales in Pregnancy: Maternal Morbidity and Perinatal Outcome," International Journal of Gynecology \& Obstetrics, Vol. 59, No. 2, 1997, pp. 109-113.

[3] J. E. Eberhart-Phillips, P. D. Frderick, R. C. Baron, et al., "Measles in Pregnancy: A Descriptive Study of 58 Cases," Journal of Gynecology \& Obstetrics, Vol. 82, No. 5, 1993, pp. 797- 801.

[4] N. Linder, E. Tallen-Gozani, B. German, et al., "Placental Transfer of Measles Antibodies: Effect of Gestational Age and Maternal Vaccination Status," Vaccine, Vol. 22, No. 11-12, 2004, pp. 1509-1514. doi:10.1016/j.vaccine.2003.10.009

[5] Y. Nakata, T. Nakayama, Y. Ide, et al., "Measles Virus Genome Detected up to Four Months in a Case of Congenital Measles,” Acta Paediatrica, Vol. 91, 2002, pp. 1263-1265. doi:10.1111/j.1651-2227.2002.tb00140.x

[6] S. Lopes, L. Rehder, P. Rota, et al., "Congenital and Neonatal Measles during an Epidemic in Sao Paulo, Brazil in 1997,” The Pediatric Infectious Disease Journal, Vol. 24, No. 4, 2005, pp. 377-378. doi:10.1097/01.inf.0000157220.00454.24

[7] G. Ohji, H. Satoh, H. Satoh, et al., "Congenital Measles Caused by Transplacental Infection,” The Pediatric Infectious Disease Journal, Vol. 28, No. 2, 2009, pp. 166-167. doi:10.1097/INF.0b013e31818a5d83 